\section{P-81 'PLACE BONDING'- A NEW MODEL TO UNDERSTANDING PARENTAL DECISION MAKING WHEN ACCESSING A HOSPICE}

1,2Helena Dunbar. ${ }^{1}$ De Montfort University, Leicester, UK; ${ }^{2}$ Rainbows Hospice for Children and Young People, Loughborough, Leicestershire, UK

10.1136/bmjspcare-2018-hospiceabs.106

Respite/short breaks have been recognized as an important supportive service in paediatric palliative care (Knapp \& Contro, 2009; Ling, 2012). In some countries, for example the UK, hospices are key players in this delivery. Despite evidence that supports short break services in hospices (Kirk \& Pritchard, 2012) the literature suggests that there is varied access and uptake of such provision (Smith, Graham, \& Herbert, 2017; Devanney \& Bardley, 2012; Knapp, Shenkman, Marcu et al., 2009). A two-phase grounded theory study was conducted in one region in England, to explore parental experiences and perceptions of children's hospices, the barriers and facilitators to access and the characteristics which parents wanted from hospice services.

A two phase grounded theory study (Charmaz, 2014) was conducted: Phase 1 used focus groups to collect data from parents of children already accessing the hospice $(n=24)$ and in Phase 2 semi-structured interviews were conducted with parents of children who did not use the hospice $(n=7)$ and parents who had previous experience of using a hospice $(n=7)$. Analysis of the data identified three main themes: Coming 'Home'; This is Living Now; and Moving Forward. A grounded theory model of place bonding was developed which illustrates the cognitive journey and characteristics which are important for parents of children with life-limiting conditions when considering/receiving hospice care for their child. Finding a place where they belonged and felt at 'home' made the decision to accept help in caring for their child with a life-limiting condition more acceptable.

\section{P-82 LEARNING DISABILITY NURSES (RNLDS) IN CHILDREN'S HOSPICES. CONTRIBUTORS OR GAME CHANGERS?}

Sabine Schwaebisch, Rhian Simmons, Emily Molony. Helen and Douglas House, Oxford, UK

\subsection{6/bmjspcare-2018-hospiceabs. 107}

Background We want to explore the versatility of RNLDs. Why and how have they become important partners in paediatric palliative care? Factors influencing this new trend (Brigham, Atkinson, Jackson et al., 2000). (i) National (children's) nursing shortage (especially in paediatric palliative care (Munn, 2017). (ii) Advancement in medical practice and technology, prolonging lives and increasing complexity of needs in children with medical conditions and disabilities (Evans, 2018). (iii) RNLDs' unique passion for supporting the most vulnerable children, and increasing motivation to advance careers in this highly specialised field.

Aim Identifying and acknowledging RNLDs' contribution to paediatric palliative care and further developing their roles and skills in hospice settings.

Method Two case studies identifying best practice examples. Collective reflection by hospice-based RNLDs on experiences and learning. Systematic analysis of RNLDs' unique skills. Map these against best practice NICE guidelines (National
Institute for Health Care Excellence, 2016). Identify competencies necessary to make PPC a safe endeavour for RNLDs. Short survey: RNLD workforce in UK children's hospices. Experiences mirrored in other children's hospices (Schwaebisch, 2018, unpublished report).

Findings RNLDs' specialised skills can contribute to making care of children with life limiting conditions safer in key areas, including enhanced communication, management of epilepsy, acute deterioration and challenging behaviour.

These and other specialised skills can make all the difference when building therapeutic relationships with families, because they include being acutely sensitive and responsive to the needs of children with profound disabilities or critical illnesses. There are moments when advocacy for the most vulnerable can have a vital impact on holistic outcomes.

Conclusion Further exploration/research in this area is needed. The evidence collected suggests RNLDs have a positive impact within the field of paediatric palliative care. For RNLDs, paediatric palliative care is an enriching and satisfying career choice. We also suggest that the palliative field invite RNLDs as nursing partners to offer people with learning disabilities the specialist person-centred care they deserve.

\section{P-83 A COLLABORATIVE PATHWAY FOR MALIGNANT PLEURAL EFFUSION}

${ }^{1}$ Suzanne Ford-Dunn, ${ }^{2}$ Kate Steele. ${ }^{1}$ St Barnabas House, Worthing, UK; ${ }^{2}$ Western Sussex Hospitals NHS Foundation Trust, Worthing, UK

\subsection{6/bmjspcare-2018-hospiceabs. 108}

Introduction For patients with known malignancy, development of malignant pleural effusion (MPE) is a poor prognostic marker: median survival with effusion at presentation is five months (Zamboni et al., 2015), but for the majority of those developing MPE during active cancer management, prognosis is much worse.

Management of pleural fluid in this setting should be individualised, depending on symptoms, functional status, prognosis and fully informed patients preferences. Pleural drainage whether single aspiration, short term drain or semi-permanent drain are all highly invasive procedures with risks attached.

Locally, patients developing MPE were frequently referred to the respiratory service for insertion of an indwelling drain inappropriately (either too unwell, or single drainage preferable, or without understanding their prognosis) and were often not known to palliative care services.

Aim To create a pathway ensuring patients developing MPE had a holistic palliative care assessment and creation of an effusion management plan appropriate to their symptoms, prognosis and informed preferences.

Method Patients developing MPE are referred to the respiratory consultant and advised they will have a palliative care review as part of the assessment process. A hospice palliative care CNS who has undertaken additional training on assessing these patients, visits at home within five days - assesses palliative care needs, discusses prognosis and options for management (semi-permanent drain/single drainage/medical management of breathlessness) with patient/family. If drainage is appropriate, the palliative care CNS liaises with the respiratory team and the patient is booked in, as well as arranging on-going palliative care support. 